\title{
3D Microelectrode Geometry Effects the Multilayer Dense Osteo Intra-organelle Membrane Potential Characterization
}

\author{
S. Sarkar \\ AEI \\ SMIT, Sikkim \\ India
}

\author{
R. Mahapatra \\ ECE \\ NIT, Durgapur \\ India
}

\begin{abstract}
We report the computational simulation study for the characterization of multilayer dense osteoblast intra organelle membrane potential in different microelectrode. The response of a cell model at various frequencies and the effect of cell parameters, such as cell membrane resistance and capacitance, were studied. We show that at low frequencies - the intra organelle can be electro porated while at high frequencies, the induced potential can be much lower than that at low frequencies at same applied voltage for dense osteo cells .we also find out that the induced TMP of osteoblast cell depends not only on its radius and geometry of the microelectrode but also the resistances and capacitances of suspending medium, which effects the dielectric property of osteoblast cell.
\end{abstract}

\section{Keywords}

Dense cell, Osteoblast cells, Simulation, Electroporation, cytoplasm, Nucleolus, Frequency Response, Intra-organelle potential.

\section{INTRODUCTION}

Osteoblasts are living biological cell containing single nucleus, responsible for bone formation. They are the most abundant and fundamental elements in bone, and their concentration in serum is closely linked to bone metabolism and serves as a biological marker for the clinical assessment of bone disease. For clinical investigation the dielectric property of osteoblast cell plays an important role. Like other biological cell the dielectric properties of osteoblast cells are very remarkable. They typically display extremely high dielectric constants at low frequencies, falling off in more or less distinct steps as the excitation frequency is increased. Their frequency dependence permits identification and investigation of a number of completely different underlying mechanisms, and hence, dielectric studies of osteomaterials have long been important in electrophysiology and biomedical application. In drug delivery system the potential of the membrane plays an important role to control the radius and density of generated pores which allows the drugs enter into the cell. This concept is also applicable for clinical application in bone cell especially in osteoblast cell.

From various research articles, it has been reported that the electric field strengths of suitable magnitudes and durations cause the biochemical and physiological changes in biological cells and tissues. When an electric field is applied to a cell or cell system, a uniform transmembrane potential (TMP) is induced on exposed cells. If the induced TMP is large enough, i.e., above the threshold value ,the cell membrane becomes permeabilized in a reversible process called electro permeabilization, thus allowing entrance of molecules that otherwise cannot easily cross the cell membrane [1]-[3]. Further increase of the electric field causes irreversible membrane permeabilization and cell death. Induced
TMP in biological cells exposed to electromagnetic fields is of interest in a variety of applications, such as gene transfect ion[4], electro chemotherapy [5], the study of forces on cells undergoing fusion, models of cardiac tissue response to defibrillating currents, and the study of potential health effects of electric and magnetic fields [6], [7]. Therefore, investigation of induced potential distribution on the osteo cell membrane is important in studying the effects of the electric field on that cell. Potential distribution on the surface of a cell placed in an electric field can be calculated analytically or numerically. Even though analytical solutions are possible only for some analytically defined shapes, such as spheroids [8], it gives us a rough picture of the dependence of the induced TMP on electric and geometric parameters. In the spherical coordinate system, a cell can be represented by a sphere surrounded with a shell as shown in fig. 1 .

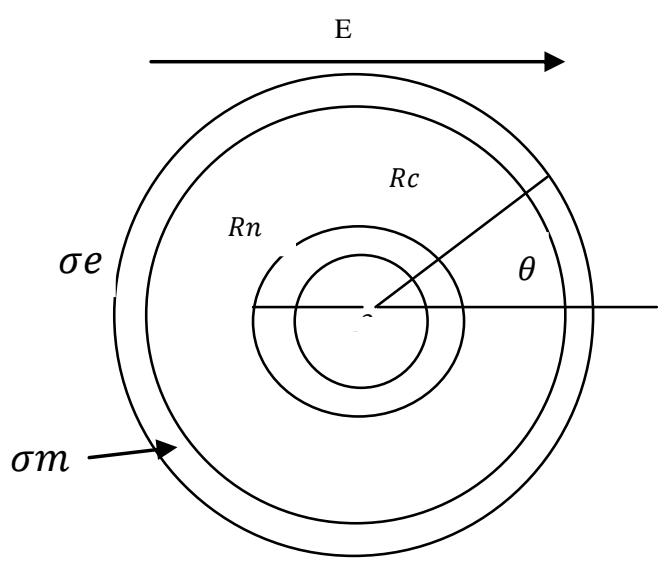

Fig. 1 spherical Cell model of osteoblast cell.

In fig. 1, we assume the cross section of a cell as a sphere, where $\sigma m, \sigma i$ and $\sigma e$ represent the specific conductivity of membrane, internal medium and external medium respectively. $\mathrm{R}$ denotes radius of cell, $d$ represents thickness and $\theta$ is the angle between electric field vector and the vector from the cell centre to any point on its surface.

Schwan explore that wwhen a voltage pulse is applied to biological cell, a charges is accumulate at the plasma membrane of it. If electric field strengths of suitable magnitudes and durations cause molecules and molecular organizations such as membranes to undergo structural rearrangements. Depending upon the magnitude and the duration of the voltage of the field applied, the cell membranes can temporarily breakdown creating pores that can be resealed eventually. The membrane potential (TMP) is given by 


$$
\begin{gathered}
T M P=1,5 E R \cos \theta /[(1+R G m(\rho i+0.5 \rho a))(1 / i \omega T))\rfloor \\
--(1)
\end{gathered}
$$

where $\rho i$ and $\rho a$ are resistivity inside and outside the cell, $\mathrm{R}$ is the cell radius, and $\theta$ is the angle between the electric field $\mathrm{E}$ and the radius vector, $\omega$ is the radian frequency $=2 \pi f$; where $f$ is the frequency, $\mathrm{T}$ is the time constant of the membrane, and $\mathrm{Gm}$ is the membrane conductance. If we assume that the cytoplasm and medium are purely resistive, then the influence of their dielectric constants can be neglected. If the conductance is also neglected, then the membrane potential is given as

$$
T M P=1.5 E R \cos \theta /(1+j \omega T)
$$

The magnitude of the equation (2) is

$$
T M P=1.5 E R \cos \theta /\left(1+(\omega T)^{2}\right)^{\frac{1}{2}}
$$

In the above equation the most important component is time constant of the membrane which is expressed as

$$
T=\frac{\epsilon m}{\left(\frac{d}{R}\right) \frac{2 \sigma e \sigma i}{2 \sigma e+\sigma i}}+\sigma m----(4)
$$

Where $\varepsilon m$ is the permittivity of the membrane. The value of $T$ is placed in equation (3) we obtain final equation as:

$$
T M P=\frac{1.5 E R \cos \theta}{\left[1+\omega\left[\frac{\epsilon m}{\left(\frac{d}{R}\right) \frac{2 \sigma e \sigma i}{2 \sigma e+\sigma i}}+\sigma m\right]^{2}\right]^{\frac{1}{2}}}----(5)
$$

when the applied electric fields are of such a magnitude so as to induce a membrane voltage of about $(0.6-1.2) \mathrm{V}$ at low frequency, the membrane becomes permeable to macro molecules otherwise it would be impermeable [10-13]. Although it is possible to manipulate osteo cells with the application of electric fields for practical use, their behaviour under electrical conditions is not completely understood. This might be due to their extremely complex structure [10].But our study gives all the information to make the complex structure into a new, simple and useful expose to the osteoblast cell.

On the other side the dielectric property of osteoblast cell is influenced by the amount of water trapping within the cell or release from the cell. The activity of water contribution in cell is expressed in terms of dispersion. So dispersion is also an important property of cell. In case of osteoblast cell dispersion is more complex and different in nature.

By using latest powerful simulation software tools, it is possible to explore the osteoblast cell behaviour in detail without conducting tedious experiments. The purpose of this research is to investigate the permeability and electro-p orated behaviour of the osteo cells by varying the electrical parameters of the model using an industry-standard simulation tool. This knowledge, which can be used as a complement to experimental analysis, is essential for effective manipulation of cells for practical, real life applications, such as electroporation-mediated gene therapy and enhancement of drug delivery system [12].

In part I of the present study we have developed the simple electrical equivalent circuit of multi layer osteoblast cell as a primary model for single cell induced by an external electric field. This model is used to investigate the process of generating the intra organelle transmembrane potential in a spherical single and dense cell, including the time evolution and related numerical analysis. The modelling results are compared to analytical data reported in the literature.

In the second part of our study, we have numerically and analytically calculate the intra organelle trans membrane Potential of multi layer single and dense osteoblast cell and the influence of geometry of electrode on cell arrangement and density on induced TMP distribution in a cell membrane. We have also calculated fraction of a cell surface where the induced TMP is above the threshold value for different cell densities and the effect of suspending medium resistances and medium capacitances on induced intra organelle voltage. All the information of our study gives the new aid to the osteo clinical diagnosis and bone cancer treatment.

\section{THEORY}

\subsection{Electrical model of multilayer osteoblast cell}

For simplified analysis of the system, an electrical circuit is often used which was developed by Foster and Schwan in 1989.The cell is approximated to a resistor that describes the cytoplasm in series with a capacitor for membrane. The cell membrane resistance is generally greater than the reactance and it is ignored. Similarly the capacitance is also ignored. After simplification we use those equations to explore the electrical model of osteoblast cell, collected from ref [29], [30] which are expressed as given below

$$
\begin{aligned}
& \text { Medium resistances }=\mathrm{Rm}=\frac{1}{\sigma \mathrm{m}(1-1.5 \mathrm{fi}) \mathrm{Gf}}---(7) \\
& \text { Medium capacitances }=\mathrm{Cm}=\frac{\epsilon \mathrm{m}(1-3 \mathrm{fi})(\mathrm{gm}-\mathrm{ei})}{2 \mathrm{\epsilon m}+\mathrm{i}}--(8) \\
& \text { Membrane capacitances }=\mathrm{Cmem}=\frac{9 \mathrm{fiRmemGf}}{4 \mathrm{~d}}---(9) . \\
& \text { Cytoplasm resistances }=R i=\frac{4\left[0.5 \sigma m+\frac{1}{\sigma m}\right]}{f i G f}--(10)
\end{aligned}
$$

$\mathrm{Gf}=\left(\frac{2 \mathrm{~d}^{3}}{3 \mathrm{D}^{2}}\right)\left(\frac{1}{1}\right)$ Whree $\mathrm{d}=$ thickness of Membrane, $\mathrm{D}=$ diameter of cell,

$\mathrm{R}=$ radius of cell, $\mathrm{l}=$ length of electrode. $\mathrm{fi}=$ area of electrode/electrode gap,

\subsection{Induced intra organelle TMP in single and dense osteoblast cell.}

Equation (1) and (2) is a very good approximation for a single spherical cell under physiological conditions, but for very low conductive external media, a more general expression has to be used[15] [9]. An analytical solution can also be derived for spheroid cells, whereas for arbitrary shaped cells, only numerical methods enable calculations for the induced TMP [18]

Schwan [9] was the first to derive the solution for a spherical cell exposed to ac electric field the membrane potential is expressed as

$$
T M P=1.5 R E \cdot \frac{1}{1+J \omega \tau}----
$$

Where time constant $\tau$ depends on the electric and geometric properties of a cell. The time constant $(\tau)$ represents a typical time needed for charging of the cell membrane and angle between electric field vector and the vector from the cell centre to any point on its surface is $\frac{\pi}{2}$. For a cell exposed to dc electric pulses of duration shorter than $\tau$ the cell interior is also exposed to the electric field, resulting in the induced trans membrane voltage across the membrane of the cellular organelles. Thus, for very short high-voltage pulses (nanoseconds), cell organelles can also be permeabilized [18], [19]. The membrane potential 
depends on the electric field which is controlled by the geometry of microelectrode as Poison's equation:

$$
E=-\nabla V-----(12)
$$

For dense cell we consider the Maxwell effect which is dedicated to the effective conductivity of particle. The calculation of the effective conductivity of a heterogeneous medium is numerically a complex problem due to the mutual interactions between the particles. A general concept incorporates all mutual interactions between the particles, with each particle being exposed to a local multi pole field which is a sum of all multi pole fields of other particles. Since this problem usually cannot be solved, different analytical EMTs which use an average "effective" field were derived [20]-[22], [23]-[26]. For calculation of the effective properties of biological systems, Maxwell mixture equation [23], [26] is most frequently used.

Maxwell derived his mixture equation for the effective conductivity $\sigma$ of a dilute suspension based on a simple example. According to Maxwell the effective conductivity is as given bellow

$$
\frac{\sigma e-\sigma}{2 \sigma e+\sigma}=f \frac{\sigma e-\sigma p}{2 \sigma e+\sigma p}----
$$

Where $\sigma \mathrm{e}$ is medium conductivity and $\sigma p$ is individual cell conductivity. $f$ Is the volume fraction of cell. According to Bruggeman formula [27]

$$
\frac{\sigma-\sigma p}{\sigma e-\sigma p}\left(\frac{\sigma e}{\sigma}\right)^{1 / 3}=1-f-----(14)
$$

The above equation however, is again an approximation that, only for certain conditions, represents a better approximation from Maxwell equation. Furthermore, for a special case of heterogeneous medium with spherical particles arranged in an SCC lattice, Rayleigh obtained the following result [27]:

$\sigma=\sigma e\left(1+\frac{3 f}{\frac{\sigma p+2 \sigma e}{\sigma p-\sigma e}-f-a \frac{\sigma p-\sigma e}{\sigma p+0.75 \sigma e} f^{10 / 3}}\right)$

Where a is a numerical factor which, according to Rayleigh, is 1.65. Later, Tobias and Meredith, following the same procedure, obtained the same formula with the corrected value of the numerical factor a being 0.523 instead of 1.65 [20], [21].

The effective conductivity causes the change of membrane potential which explore in the derivation of Qin et al [29].According to this the analytical approximation for the frequency-dependent induced TMP on dense cells arranged in cubic lattices as follows

$$
T M P=\left(\frac{1.5 E 0(j \omega) R \cos \theta}{1+\left(\frac{3 f}{4 N \pi}\right)^{\frac{1}{3}}}\right)\left(\frac{1}{1+j \omega}\right)----(1
$$

Where $f=\frac{4 \pi}{3} N\left(\frac{R}{A}\right)^{3}$ and where $N$ is the number of spheres contained in each unit cell: $\mathrm{N}=1(\mathrm{sc}), \mathrm{N}=2$ (bcc), and $\mathrm{N}=4$ (FCC). From geometries of the unit cells, it follows that the maximum volume fractions for solid spheres are $0.52,0.64$, and 0.74 for sc, bcc, and FCC lattices, respectively. As osteoblast cell is arranged in FCC lattices structure at the surface portion of bone cell so we consider the equation (16) for analytical \& simulation study of dense osteoblast cell.

\subsection{Effect of medium resistances $(\mathrm{Rm})$ and of medium capacitances $(\mathrm{Cm})$ on intra organelle TMP in osteoblast cell.}

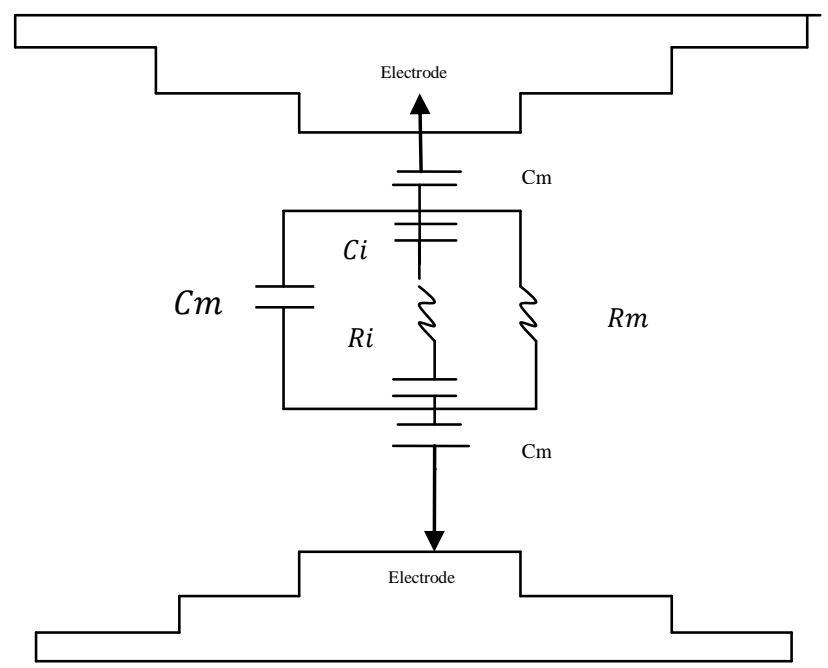

Fig. 2: diagram showing the equivalent circuit model for a single cell in suspension. $\mathrm{Rm}$ And $\mathrm{Cm}$ is the equivalent resistance and capacitance of the medium, respectively. Cmem Is the equivalent capacitance of the cell membrane? $R i$ Is equivalent resistance of the cell cytoplasm?

The values of the individual electrical components in the circuit model are determined by the cell size, dielectric properties of the medium and the cell, the volume fraction and also the geometrical parameters of the chip[30],[31].From the ref [30] \& [31] we find that,

$$
\text { medium resistances }\left(R_{m}\right)=\frac{1}{\sigma_{m}\left(1-\frac{3 \varphi}{2}\right) l k}-
$$

medium capacitances respectively $(m)$

$$
\begin{gathered}
=\varepsilon m \frac{2 \epsilon m+\epsilon i-2 \varphi(\epsilon m-\epsilon i)}{2 \varepsilon m+\varepsilon i+\varphi(\varepsilon m-\epsilon i)} l k \\
------(18)
\end{gathered}
$$

Where $k=\frac{\text { width of electrode }}{\text { inter electrode distances }}, \& l$ is the length of electrode. $\varphi$ is the volume fraction .From the above equation (17) $\&$ (18) we find out the relation of $\boldsymbol{\sigma} \boldsymbol{m} \& \boldsymbol{\varepsilon} \boldsymbol{m}$ with other parameters and put them into equation (6). As a result we find out a numerical equation which shows that the trans membrane potential (TMP) adequately depends on medium resistance \& capacitances.

\section{SIMULATION TOOL}

For fast and accurate simulation results, we chose the semiconductor, RF, and industry preferred NI Circuit Simulator and lab-view, product version (2.5.1).Lab view is commonly used for complex analogue and mixed-signal circuits .In an application, NI Circuit Simulator is used to study the circuit design .The analytical and mathematical simulation is done by the latest version MATLAB 10.5 and Math AutoCAD. Similarly, the electric field analysis of various electrodes is done by COMSOL metaphysics software. 


\section{PARAMETER VALUE USED:}

In our study we used hybrid micro chip whose top view and potential distribution are shown in fig: $3 \& 4$. Specification and generated electric field intensity of the used microelectrodes are explore in fig 4, where as the values are referred in table I \& II respectively.

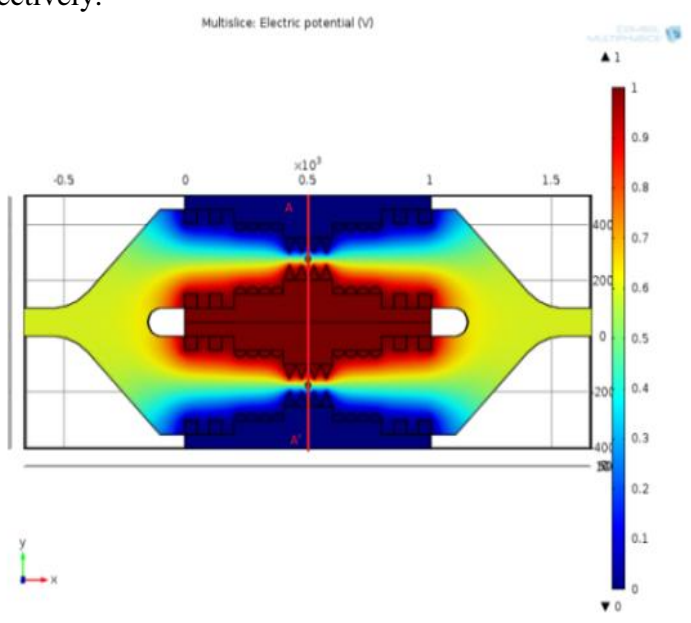

Fig 3 Top view of electrical potential distribution within the 3D hybrid micro chip.

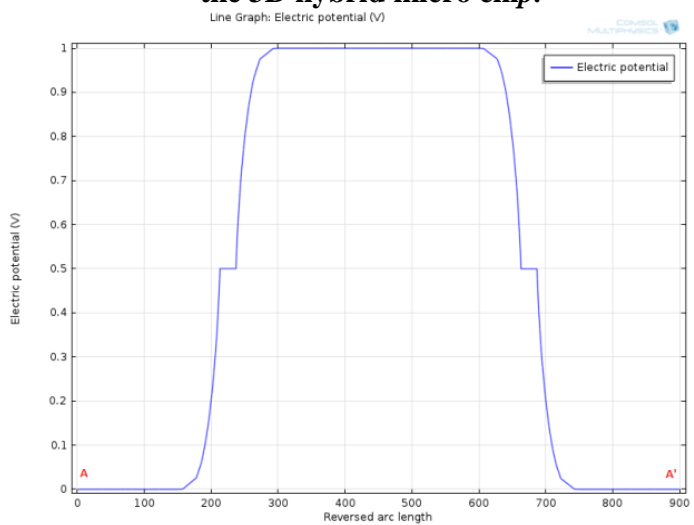

Fig 4 Graphical view of electrical potential distribution with respect to height of the bi metallic electrode within the 3D hybrid micro chip.

Table-1 Electrode specification

\begin{tabular}{|l|l|}
\hline Length of electrode (l) & $1 \mathrm{~mm}$ \\
\hline Width of electrode $(\mathrm{w})$ & $100 \mu \mathrm{m}$ \\
\hline Inter-electrode distance & $100 \mu \mathrm{m}$ \\
\hline Thickness of electrode(d) & $1 \mu \mathrm{m}$ \\
\hline$\varepsilon_{0}$ & $8.856 \times 10^{-12} \mathrm{~F}$ \\
\hline
\end{tabular}

TABLE- 2 ELECTRIC field intensity calculating from comsole multi physics s/w [49].

\begin{tabular}{|c|c|c|}
\hline Type of electrode & $\begin{array}{c}\text { Rotation of } \\
\text { electrode }\end{array}$ & $\begin{array}{c}\text { Electric field } \\
\text { intensity(10) }\end{array}$ \\
\hline \multirow{2}{*}{ Rectangular } & In line(c) fig:4 & 3.68 \\
\cline { 2 - 3 } & Shift (d) fig:4 & 3.75 \\
\hline \multirow{2}{*}{ Semicircular } & $\begin{array}{c}\text { In line (a) } \\
\text { fig:4 }\end{array}$ & 4.22 \\
\cline { 2 - 3 } & Shift (b) fig:4 & 4.07 \\
\hline \multirow{2}{*}{ Saw tooth } & In line(e) fig:4 & 8.87 \\
\cline { 2 - 3 } & Shift (f) fig:4 & 3.61 \\
\hline Co-centric & & V/log(b/a) \\
\hline
\end{tabular}

TABLE-3 USED numerical value of osteoblast cell

\begin{tabular}{|c|c|}
\hline PARAMETERS & Single osteoblast cell \\
\hline $\operatorname{Radius(R)}$ & $12 \mu m$. \\
\hline Thickness(d) & $0.006 \mu \mathrm{m}$ \\
\hline Cytoplasm conductivity ( $\sigma \mathrm{i})$ & $0.039 \mathrm{~s}$ \\
\hline Medium conductivity $(\sigma \mathrm{m})$ & $10 \times 10^{-3} \mathrm{~S}$ \\
\hline Membrane conductivity $(\sigma \mathrm{mem})$ & $1.2 \times 10^{-7}$ \\
\hline Cytoplasm permittivity( $(\varepsilon i)$ & $93 \varepsilon_{0}$ \\
\hline Medium permittivity $(\mathrm{\varepsilon m})$ & $80 \varepsilon_{0}$ \\
\hline Membrane permittivity(smem) & $22 \varepsilon_{0}$ \\
\hline First relaxation time & $1.198 \times 10^{-9} \mathrm{sec}$ \\
\hline Second relaxation time & $57.12 \times 10^{-9}$ sec. \\
\hline
\end{tabular}

\section{RESULTS AND DISCUSSION} 5.1 Electrical model of multi layer osteoblast cell.

For numerical and analytical calculation we use Mat lab and NI lab view and the electrode which is used in this simulation is specified as Table-I.

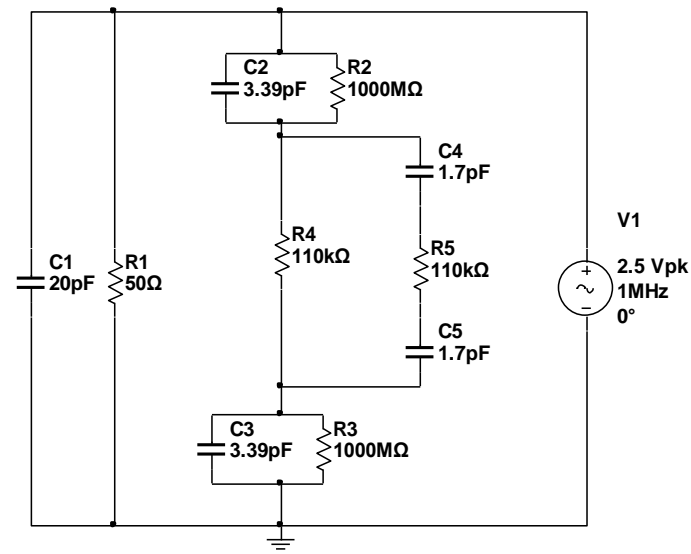

Fig.5 Electrical model of multi layer osteoblast cell.

For this simulation study, a slightly modified version of the more detailed electrical model of a cell reported by Achenbach et al. 
was used [3]. Fig. 5 illustrates the cell model and simulated value of various cell parameters by using some unique equation [29] [30].This model is used for numerical and analytical study of osteoblast cell. Here, the cell was modelled as a homogenous conductive medium (cytoplasm) surrounded by a leaky dielectric membrane. The plasma membrane was modelled as parallel combination resistors R2 and R3, together with capacitors C2and C3.The simulated value of each register and capacitor are $1000 \mathrm{M}$ ohm and 3.39microfarad respectively which is calculated in our previous work... The interior of the cell substructure, i.e., the nucleus, was modelled as of resistors R5 and half of membrane capacitors $\mathrm{C} 4$ and C5: These nucleus model elements were connected in parallel with the R4, which represents the conductive cytoplasm. The values of the resistances and capacitances chosen for cytoplasm and nucleus psalm were estimated using a spherical cell having the diameter of 24 micro meters. The simulated value of nucleus resistances and capacitances are 110killo ohm and 1.7microfaradrespectively. The resistivities of the cytoplasm and nucleus psalm were assumed to be the same at, 100kilo ohm. The specific capacitance of the outer membrane was 3.29 microfarad. The capacitance of the nuclear membrane was assumed to be half that of the outer membrane because two lipid membranes comprise the nuclear envelope, whereas the outer membrane consists of only one [1]. Input voltages of magnitudes between 1.0 and $5 \mathrm{~V}$ and $0.5 \mathrm{~ms}$ duration were used in the numerical study of electroporation. The specification of used electrode is explores in table-I which is shown bellow. This value is also used in clinical trials. Consequently, an input voltage of $1 \mathrm{~V}$ ac was used to test and simulate the characteristics of a single osteo cell. Assuming the cell radius be $12 \mu \mathrm{m}$, the resulting field strength becomes 1 $\mathrm{kV} / \mathrm{cm}$, which is in close correlation with the value of $1300 \mathrm{~V} / \mathrm{cm}$ used in practice [50]-[54].

\subsection{Analytical study of intra organelle TMP for osteoblast cell:}

The classical theory of TMP developed by Schwan [48] leads to a temporal description of this phenomenon as a first-order process. The expression of the membrane potential define as a function of the time during the rising edge of the signal is given as

$$
V m(t)=K \frac{d V}{d t}\left(t-\tau+\tau e^{-\frac{t}{\tau}}\right)-----(19
$$

Where $\mathrm{K}$ is the $\mathrm{DC}$ gain, which is calculated as

$$
V m=\frac{3 E R}{2}, E=\frac{V p p}{d} \text { so } K=\frac{3 R}{2 d}-----
$$

Where Ethe strength of the electric is field and Vpp is the applied voltage between the two electrodes.

The analytical expression of membrane potential (TMP) given as [43]-[47].

K

$=\left(\frac{3 e \sigma 0\left(\sigma i\left(3 R^{2}-3 e R+e^{2}\right)+\sigma m\left(3 e R^{2}-e^{2}\right)\right)}{2 R^{3}(\sigma m+2 \sigma 0)\left(\sigma m+\frac{1}{2} \sigma i\right)+2(R-e)^{3}(\sigma m-\sigma 0)(\sigma i-\sigma m)}\right)\left(\frac{R}{d}\right)$ (21)

By using the numerical value given in table II \& consider radius $\mathrm{R}=12 \mu \mathrm{m} \& \sigma 0=10 \mathrm{~ms}$, we find the value of $\mathrm{K}=3.96$ by using the equation (19) \& K=4 by using (21) as the difference between them is very low, so (19) is used for analytical calculation.
To find out the influence of the slope $\frac{\mathrm{dv}}{\mathrm{dt}}$, we focus on the time ts required to reach threshold voltage Vs. Experiments show that the time during which the amplitude of the applied pulse exceeds a certain critical value, has a major role in the efficiency of electropermeabilization [48]-[49]. It is assumed here that this critical value is linked with the reversible threshold of permeabilization.

In equation (19), the exponential term are neglected, and an expression pulse duration (ts) of as a function of threshold voltage Vs and $\frac{\mathrm{dv}}{\mathrm{dt}}$ would be

$$
t s=\frac{V s}{K \frac{d v}{d t}}+\tau-----
$$

We follow the above equation and studied the variation of ts and $\frac{\mathrm{dv}}{\mathrm{dt}}$ in high \& low medium conductivity and maximum \& minimum resistances of osteoblast cell.

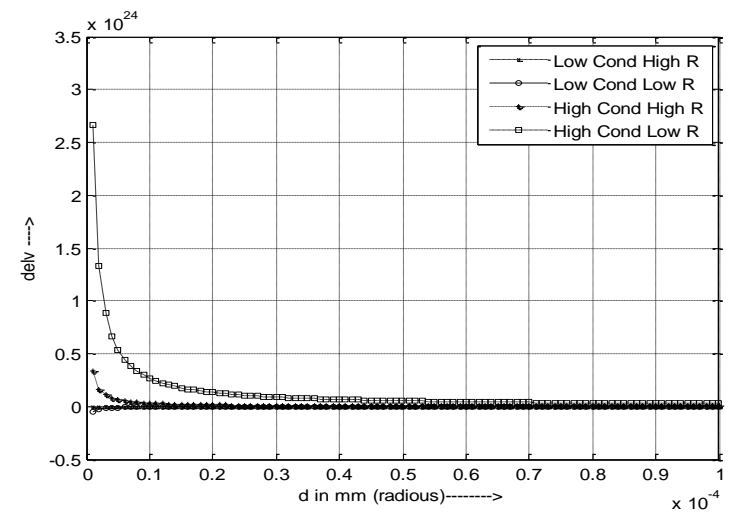

Fig. 6 ts versus $\frac{d v}{d t}$ in in high \& low medium conductivity and maximum and minimum resistances of osteoblast cell

In Fig. 6, values of $t_{s}$ is given by analytical expression in equation (21). For radius value of $12 \mu \mathrm{m}$ we obtain the different value of $\tau$ when we change the medium conductivity and radius of osteoblast cell. We also find that the accuracy of the analytical expression of $t_{s}$ improves, when the slope $\frac{d v}{d t}$ and $\tau$ present low values. We find that when the medium conductivity is high but radius is low the ts is rapidly changed with $\frac{\mathrm{dv}}{\mathrm{dt}}$ but in case of low medium conductivity and low radius the $t_{s}$ remain constant although slope $\frac{d v}{d t}$ is changed. From the above result we can conclude that the time $t_{s}$ required to reach the threshold voltage is influenced by the cytoplasm and the extracellular medium conductivities as well as osteoblast cell radius.

\subsection{CONTROL OF PULSE:}

Pulses used for electropermeabilization can be very short, especially for eukaryote cells, on which single rectangular pulses of 100- $\mu$ s duration are often applied [49].

If pulse generators able to deliver signals with slopes greater than $\frac{d v}{d t}$, otherwise the TMP would remain below the reversible threshold during a time $t_{\mathrm{s}}$ which is not negligible in comparison with the total pulse duration. Ts not are precisely controlled: it depends particularly on the dc gain and the threshold voltage Vs value, which are generally imprecisely known and present heterogeneous values. Then, for low values of $\frac{\mathrm{dv}}{\mathrm{dt}}$, could be different from a one cell to another; the effective duration of the 
applied pulses being unknown, the experiments would not be fully repeatable. On the other hand, generator pulses with slewrates much greater than seem unnecessary, at least for high values of K [49].

In our study the slope of the signal is greater than $\frac{d v}{d t}$, so TMP is greater than threshold voltage.

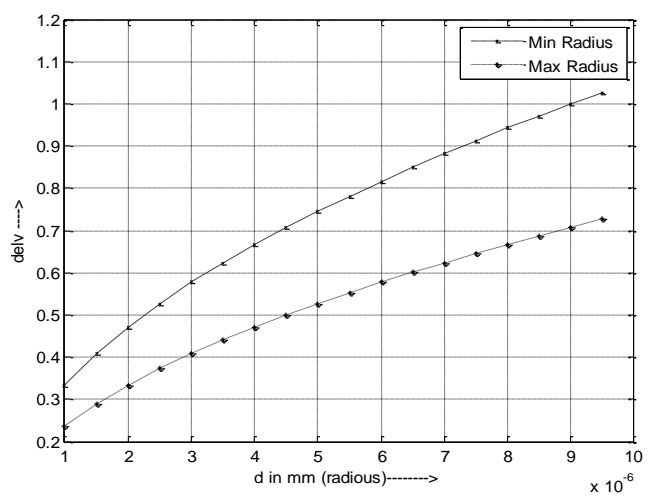

Fig . $7 \frac{\mathrm{dv}}{\mathrm{dt}}$. Versus distance between two electrodes

Fig. 7 shows values of the $\frac{d v}{d t}$ in case of osteoblast cells with a radius $\mathrm{R}=12 \mu \mathrm{m} \& 10 \mu \mathrm{m}$ for a distance between electrodes included in $10 \mu \mathrm{m}$. it can be seen that high values of slew-rate can be required for low radius value. In this purpose special generators or reduction of the distance between electrodes may be necessary.

\subsection{Calculation and variation of intra organelle transmembrane potential for single and dense osteoblast cell in different microelectrode.}

\subsubsection{Single cell}

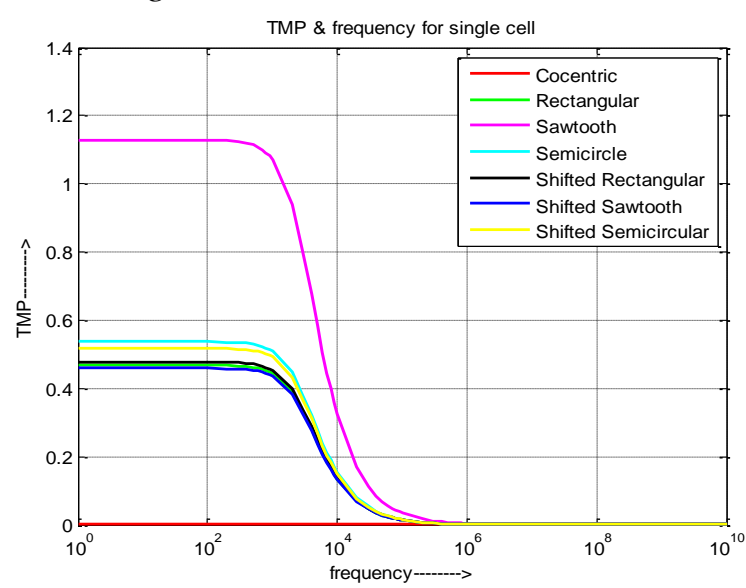

Fig. 8 (a) Variation of intra organelle trans-membrane potential (TMP) of single osteoblast cell with frequency over different type of electrode

With the help of the equation (6) and simulated value shown in table-II, we plot the curbs (fig 8a and 8b) which explore the variation of the Trans membrane potential of single $\&$ dense osteoblast cell with frequency over different type of electrode. It also explore that the induced transmembrane potential in an Osseo cell depends upon the magnitude, and duration (frequency) of the applied electric field as well as geometry of electrode. We studied this phenomenon by varying the frequency of the applied voltage and by performing an ac analysis over different type of electrode.

Fig8 (a) show the potentials induced across the membrane of osteo cell as functions of frequency. It exhibits low-pass filterlike characteristics with the cut off frequency occurring at about $\mathrm{kHz}$ to $\mathrm{MHz}$ depending on the geometry of the microelectrode. From fig 7(a) the maximum and minimum membrane potentials are obtained for saw tooth and shifted sawtooth electrodes respectively. In this figure we also observed that if the frequency is increased, the voltage output at the cell membrane decreased approaching a very small value near $\mathrm{GHz}$ range. At low frequencies of applied voltage, the membrane, which has a large capacitance, is affected. At these frequencies, the induced voltage is varied from 0.45 volt to 1.128 volt, which is greater than the threshold voltage for electro proration. It also explores that the time required for pore formation and pore density is the function of structure of micro electrode. On the other hand it also reveals that membrane potentials at microwave frequencies are therefore much smaller than at lower frequencies. Hence, membrane interactions are less likely to occur at such high frequencies. At very high frequencies, the membrane capacitance sustains almost no voltage drop, and the total voltage applied to the cell is available to the cytoplasm. This phenomenon was simulated using the present electrical model of osteo cell.

\subsubsection{Dense Cell}

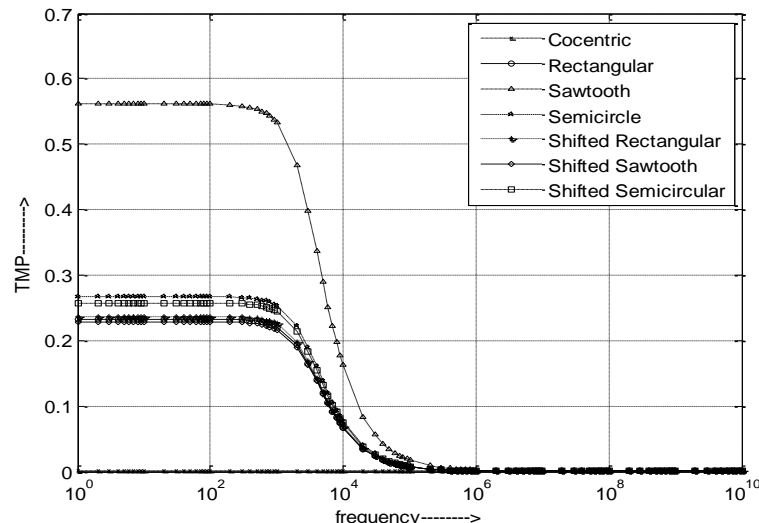

Fig: 9 (b) Variation of intra organelle Trans membrane potential (TMP) of cluster osteo cell with frequency over different type of electrode.

Fig9 (b) shows variation of transmembrane potential (TMP) of cluster osteo cell with frequency over different type of microelectrode. We observed that the TMP value is reduced for dense osteoblast cell compare with single cluster cell having the same frequency and shape of microelectrode. This result concludes that the cluster of osteoblast cell is more rigid than single osteoblast cell and it needs higher electrical pulse to electro orate dense osteoblast cell.

\subsection{Effect of medium capacitances on intra organelle transmembrane potential in various microelectrode.}




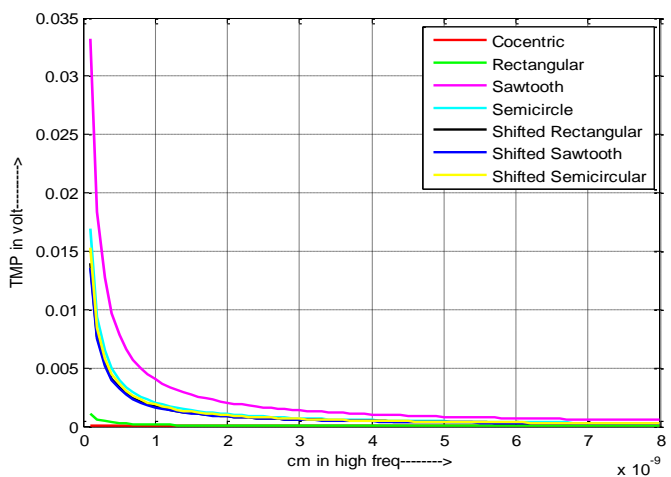

(a)

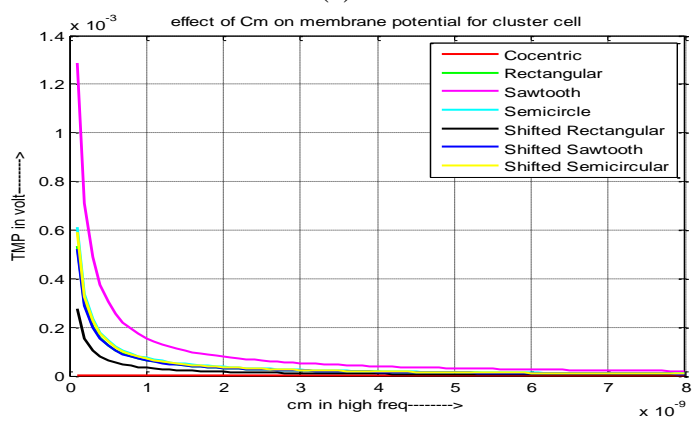

(b)

Fig10: Variation of intra organelle transmembrain potential (TMP) with medium capacitances over different type of electrode at high frequency. (a) Single cell, (b) cluster cell

We have studied the effect of changing the medium resistance and capacitance at very high $(1 \mathrm{GHz})$ and very low $(50 \mathrm{~Hz})$ frequencies as shown in figs10-13. Fig: 10 show the change in the induced membrane potential when the medium capacitance was changed from $100 \mathrm{pF}$ to $100 \mathrm{NF}$ at high frequency over different type of electrode. There is an exponential decay of voltage induced across the membrane. There is not a very significant effect in this case because the membrane voltage is very small at high frequencies.

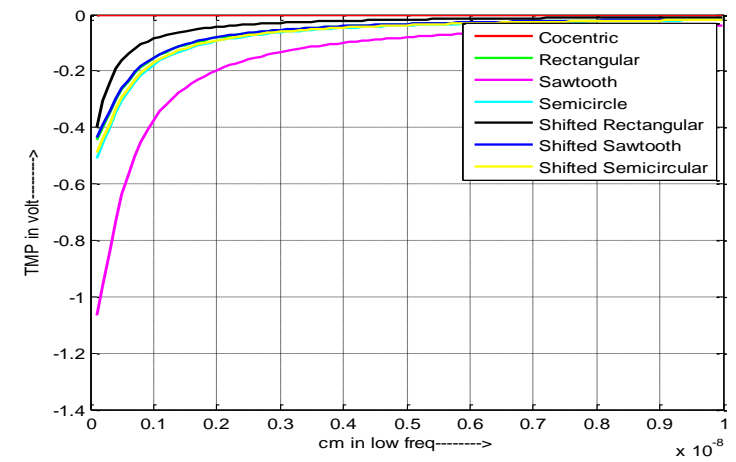

(a)

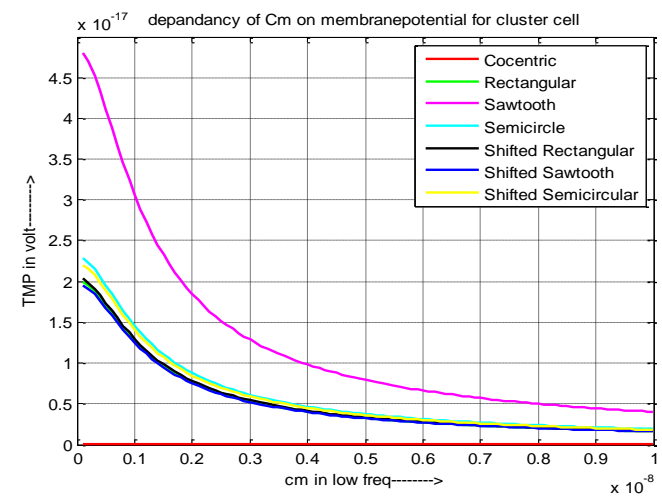

(b)

Fig 11 Variation of intra organelle transmembrane potential (TMP) with medium capacitances over different type of electrode at low frequency. (a) Single cell, (b) cluster cell

Fig 11 shows the noticeable effect obtained in the case of a low frequency of $100 \mathrm{~Hz}$ when the medium capacitance was changed from $100 \mathrm{pF}$ to $100 \mathrm{nF}$. We noted the significant difference in membrane potential values in different type of microelectrode such as the max value of TMP for saw tooth electrode is1.1 Volts, for rectangular electrode is $0.5 \mathrm{v}$ and for semicircular electrode is $0.55 \mathrm{v}$.so better output is obtain in saw tooth electrode.

\subsection{Effect of medium resistances on intra organelle transmembrane potential in various microelectrode.}

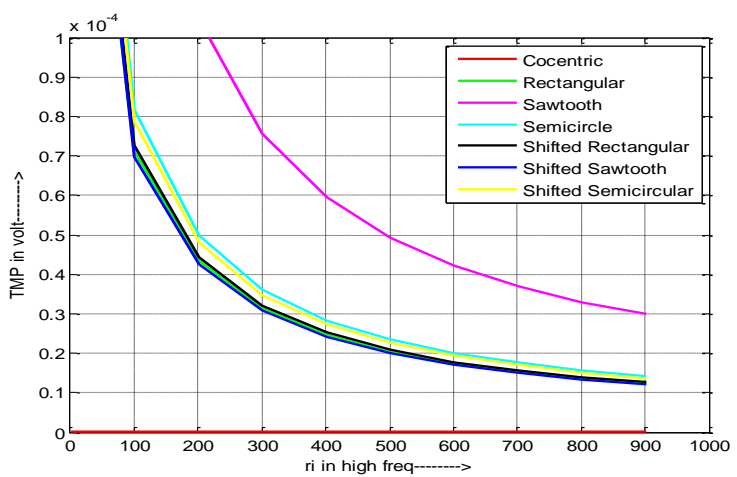

(a)

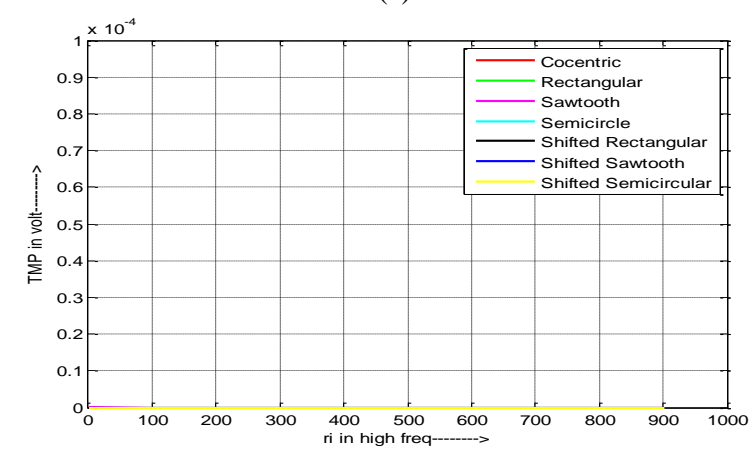

(b)

Fig: 12 Variation of intra organelle Trans membrane potential (TMP) with medium resistances over different type of electrode at high frequency. (a) Single cell, (b) cluster cell

Fig:12, shows the results when the medium resistance was varied from $10 \Omega$ to $1 \mathrm{k} \Omega$ at $1 \mathrm{GHz}$, an exponential decrease occurs in the voltage measured at the output for different type of electrode. There is an exponential decay of voltage induced across the 
membrane. There is not a very significant effect in this case because the membrane voltage is very small at high frequencies.

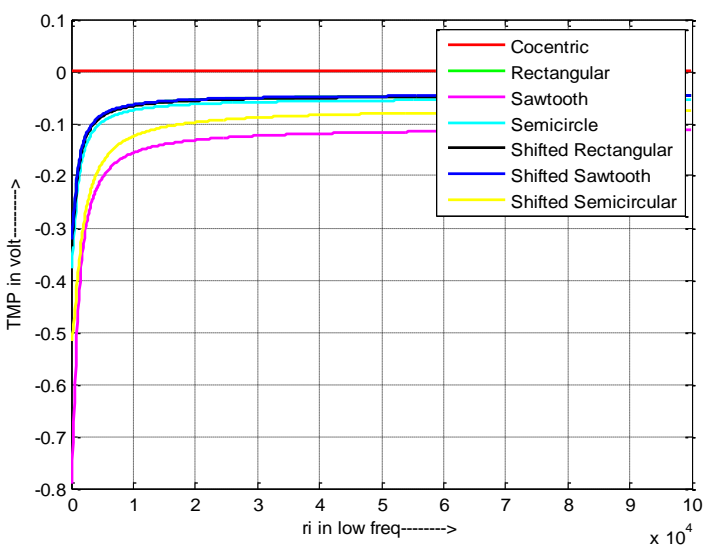

(a)

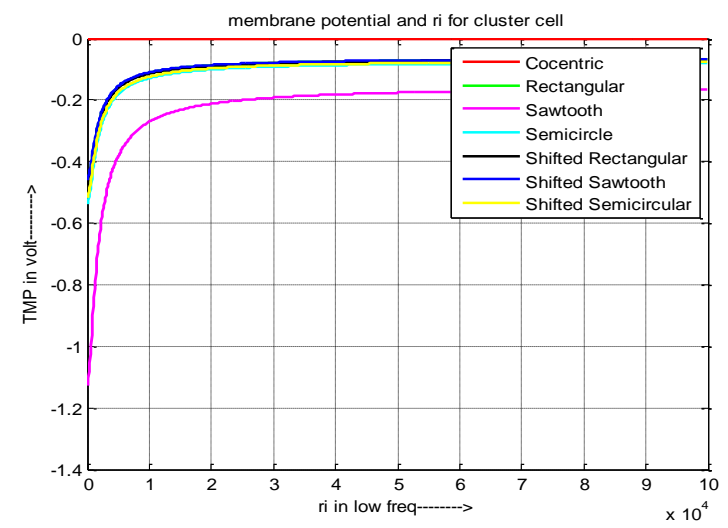

(b)

Fig: 13, Variation of intra organelle Trans membrane potential (TMP) with medium resistances over different type of electrode at low frequency. . (a) Single cell, (b) cluster cell

Fig. 13 shows the results when the medium resistance was varied from $10 \Omega$ to $10 \mathrm{k} \Omega$ at $1 \mathrm{GHz}$. An exponential increase occurs in the voltage measured at the output, with the membrane potential in the order of (0.2-1) V. Compare these results with those obtained at a low frequency of $200 \mathrm{~Hz}$ (Fig. 11) for the same medium resistance change as above. This result due to the increased of voltage causing a dielectric breakdown to occur more easily at higher resistivity than at lower resistivity in osteoblast cell.

\section{SUMMARY}

The electrical properties of osteoblast cells are extremely complex. They behave differently at various frequencies over different type of micro electrode. Electrical modelling can be effective in studying the ac frequency response of osteoblast cells. The electrical model serves as a great aid to understanding the behaviour of single osteo and cluster osteo cells under over a wide frequency range. The results confirm that a high frequency, alternating electric field might also be used for electroporation for osteoblast cell .It also provides the new data electroporation of the same cell. This study reveals that in intra organelle osteoblast cell transmembrane potential characterization, density \& radius of pore in membrane can be controlled by changing the microstructure of electrode. All these are related to the dielectric properties of the osteoblast cell which can also aid in understanding the basic physiological difference between normal and cancerous cells on a molecular level. More work has to be done to explore these possibilities.

\section{REFERENCES}

[1] L. M. Mir, "Therapeutic perspectives of in vivo cell electropermeabilization," Bioelectrochemistry, vol. 53, pp. $1-10,2000$.

[2] E. Neumann, A. E. Sowers, and C. A. Jorda, Electroporation and Electrofusion in Cell Biology. New York: Plenum, 1989.

[3] S. Orlowski and L. M. Mir, "Cell electroporation: A new tool for biochemical and pharmacological studies," Biochim. Biophys. Acta, vol 1154, pp. 51-62, 1993.

[4] L. M. Mir,M. F. Bureau, J. Gehl, R. Rangara, D. Rouy, J.M. Caillaud, P.Delaere, D. Branellec, B. Schwartz, and D. Scherman, "High-efficiencygene transfer into skeletal muscle mediated by electric pulses," in Proc.Nat. Acad. Sci. USA, vol. 96, 1999, pp. 4262-4267.

[5] G. Sersa, T. Cufer, M. Cemazar, M. Rebersek, and Z. Rudolf, "Electrochemotherapy with bleomycin in the treatment of hypernephromametastasis," Case Report and Literature Review, Tumori, vol. 86, pp. 163-165, 2000.

[6] C. E. Fear and M. A. Stuchly, "Biological cells with gap junctions inlow-frequency electric fields," IEEE Trans. Biomed. Eng., vol. 45, pp.856-866, July 1998.

[7] "Modeling assemblies of biological cells exposed to electricfields," IEEE Trans. Biomed. Eng., vol. 45, pp. 1259-1271, Oct. 1998.

[8] T. Kotnik and D. Miklav`ci cc, "Analytical description of transmembrane voltage induced by electric fields on spheroidal cells,” Biophys. J., vol.79, pp. 670-679, 2000.

[9] H. P. Schwan, "Electrical properties of tissue and cell suspensions," Adv.Biol. Med. Phys., vol. 5, pp. 147-209, 1957.

[10] H.P. Schwan, Dielectrophoresis and rotation of cell, in: E. Neumann, A.E. Sowers, C.A. Jordan (Eds.), Electroporation and Electrofusion in Cell Biology, Plenum press, New York, 1989.

[11] U. Zimmermann, G.A. Neil, Electromanipulation of Cells, CRC Press, New York, 1996.

[12] K.H. Schoenbach, et al., Bioelectrics-new applications for pulsed power technology, IEEE Trans. Plasma Sci. 30 (1) (2002).

[13] S.B. Dev, D.P. Rabussay, G. Widera, G.A. Hoffmann, Medical applications of electroporation,IEEE Trans. Plasma Sci. 28 (1) (2000) 206-223.

[14] M. Jaroszeski, R. Heller, R. Gilbert (Eds.), Electrochemotherapy, Electrogenetherapy, and Transdermal Drug Delivery: Electrically Mediated Delivery of Molecules to Cells, Methods in Molecular Medicine, vol. 37, Humana Press, Totowa, NJ, 2000.

[15] E. Neumann, A. E. Sowers, and C. A. Jordan, Electroporation and Electrofusion in Cell Biology. New York: Plenum, 1989

[16] J. C. Weaver and Y. A. Chizmadzhev, "Theory of electroporation:A review," Bioelectrochem. Bioenerg., vol. 41, no. 2, pp. 135-160,Dec. 1996. 
[17] G. Pucihar, T. Kotnik, B. Valic, and D. Miklav`cicc, "Numerical determination of transmembrane voltage induced on irregularly shaped cells," Ann. Biomed. Eng., vol. 34, no. 4, pp. 642-652, Apr. 2006.

[18] A. G. Pakhomov, J. F. Kolb, J. A. White, R. P. Joshi, S. Xiao, and K. H. Schoenbach, "Long-lasting plasma membrane permeabilization in mammalian cells by nanosecond pulsed electric field (nsPEF)", Bioelectromagnetics, vol. 28, no. 8, pp. 655-663, Dec. 2007.

[19] K. H. Schoenbach, S. Xiao, R. P. Joshi, J. T. Camp, T Heeren, J. F. Kolb, and S. J. Beebe, "The effect of intense subnanosecond electrical pulses onbiological cells", IEEE Trans. Plasma Sci., vol. 36, no. 2, pp. 414-422,Apr. 2008.

[20] S. S. Dukhin, "Dielectric properties of disperse systems in Surface andColloid Science", vol. 3, E.Matijevi'c, Ed. New York:Wiley-Interscience,1971, pp. 83-165.

[21] S. Takhasima, Electrical Properties of Biopolimers and Membranes,Bristol, U.K.: Adam Hilger, 1989.

[22] T. Hanai, "Electrical properties of emulsions", in Emulsion Science,P. Sherman, Ed. London, U.K.: Academic, 1968, pp. 353-478.

[23] L. Rayleigh, "On the influence of obstacle arranged in rectangular order upon the properties of a medium", Philos. Mag., vol. 34, pp. 481-502,1892.

[24] J. C. Maxwell, Treatise on Electricity and Magnetism. London, U.K.:Oxford Univ. Press, 1873.

[25] K. W. Wagner, "Erklärung der dielektrischen Nachwirkungsvorgänge aufGrund Maxwellscher Vorstellungen", Archiv für Elektrotechmik II, vol. 9,pp. 371-387, 1914.

[26] V. H. Pauly and H. P. Schwan, "Über die Impendanc einer Suspensionvon kugelförmigen teilchen mit einer Schale", Z. Naturforsch., vol. 14b,pp. 125-131, 1959.

[27] L. Rayleigh, "On the influence of obstacle arranged in rectangular orderupon the properties of a medium", Philos. Mag., vol. 34, pp. 481-502,1892.

[28] Y. Qin, S. Lai, Y. Jiang, T. Yang, and J. Wang, "Transmembrane voltageinduced on a cell membrane in suspensions exposed to an alternating field:A theoretical analysis", Bioelectrochemistry, vol. 67, no. 1, pp. 57-65,Sep. 2005.

[29] K. R. Foster and H. P. Schwan, Rev. Biomed. Eng.17, 25 (1989).

[30] H. Morgan, T. Sun, D. Holmes, S. Gawad and N. G.Green, J. Phys. D: Appl. Phys. 40, 61 (2007)

[31] Schwan, H.P.; Takashima, S. Electrical conduction anddielectric behavior in biological systems. Encycl. Appl. Phys. 1993, 5, 177-200.

[32] Fricke, H. The complex conductivity of a suspension of stratified particles of spherical cylindrical form. J. Phys.Chem. 1955, 59, 168.

[33] Foster, K.R.; Schwan, H.P. Dielectric properties of tissue.CRC Crit. Rev. Biomed. Eng. 1989, 17, 25-104.

[34] Schwan, H.P.; Morowitz, H.J. Electrical properties of themembranes of the pleuro-pneumonia-like organism A5969, Biophys. J. 1962, 2, 295.
[35] Schwan, H.P.; Takshima, S.; Miyamoto, V.K.; Stoeckenius,W. Electrical properties of phospholipid vesicles.Biophys. J. 1970, 10, 1102

[36] Kirkwood, J.G. The dielectric polarization of polar liquids.J. Chem. Phys. 1939, 7, 911.

[37] Onsager, L.J. Electric moments of molecules in liquids.Am. Chem. Soc. 1936, 58, 1486-1493.

[38] Mandel, M.; Odijk, T. Dielectric properties of polyelectrolytesolutions. Ann. Rev. Phys. Chem. 1984, 35, 75-108.

[39] Martinsen, 1.G.; Grimnes, S.; Karlsen, J. "Low frequency dielectric dispersion of microporous membranes in electrolyte solution", J. Colloid Interface Sci. 1998, 199, $107-110$

[40] Takashima, S. Electrical Properties of Biopolymers and Membranes; Adam Hilger: Bristol, 1989.

[41] Pethig, R. Dielectric and Electronic Properties of Biological Materials; Wiley: New York, 1979.

[42] Pethig, R.; Kell, D.B. "The passive electrical properties of biological systems: their significance in physiology", biophysics and biotechnology. Phys. Med. Biol. 1987, 32,933-970.

[43] Schwan, H.P.; Bothwell, T.P.; Wiercinski, F.J. "Electricalproperties of beef erythrocyte suspensions at low frequencies", Fed. Proc. Am. Soc. Exp. Biol. 1954, 13, 15.

[44] Schwan, H.P.; Bothwell, T.P. Electrical properties of the plasma membrane of erythrocytes at low frequencies. Nature $1956,178,265$

[45] Schwan, H.P.; Carstensen, E.L. Dielectric properties of the membrane of lysed erythrocytes. Science 1957, 125, 985.

[46] Fatt, P. An analysis of the transverse electrical impedance of striated muscle. Proc. R. Soc. London, Ser. B 1964, 159, 606-651.

[47] Schwan, H.P. Electrical Properties of Tissue and Cell Suspensions.In Advances in Biological and Medical Physics; Lawrence, J.H., Tobias, C.A., Eds.; Acad. Press: New York, 1957; Vol. V, 147-209.

[48] T.Kotnik, G. Pucihar,M. Rebersek, D. Miklavcic, and L. M. Mir, "Role of pulse shape in cell membrane electropermeabilization," Biochimica et Biophysica Acta, no. 1614, pp. 193-200, Aug. 2003

[49] I. Meny, N. Burais , F. Buret , and L. Nicolas "FiniteElement Modeling of Cell Exposed to Harmonic and Transient Electric Fields" IEEE transactions on magnetics, vol. 43, no. 4, April 2007.

[50] H.P. Schwan, Dielectrophoresis and rotation of cell, in: E. Neumann, A.E. Sowers, C.A. Jordan

[51] (Eds.), Electroporation and Electrofusion in Cell Biology, Plenum press, New York, 1989

[52] U. Zimmermann, G.A. Neil, Electromanipulation of Cells, CRC Press, New York, 1996.

[53] [3] K.H. Schoenbach, et al., Bioelectrics - new applications for pulsed power technology, IEEE Trans.

[54] S.B. Dev, D.P. Rabussay, G. Widera, G.A. Hoffmann, Medical applications of electroporation, Plasma Sci. 30 (1) (2002). 